\title{
Papel y perfil del docente en la transición a clases virtuales
}

\section{Role and profile of the teacher in the transition to virtual classes}

Recibido 17/04/2021

Aceptado 01/07/2021

\section{Referencia}

Orellana Linares, Y. A. (2021). Papel y perfil del docente en la transición a clases virtuales. Revista Docencia Universitaria, 2(2), 32-40. https://doi.org/10.46954/revistadusac.v2i2.29

\section{Resumen \\ Resumen}

Yeny Audelí Orellana Linares

Maestría en Docencia Universitaria

Universidad de San Carlos de Guatemala, yorellana1303@gmail.com

https://orcid.org/0000-0002-7482-5027

Este artículo presenta una descripción del papel y perfil que los docentes de la Facultad de Ciencias Económicas de la Universidad de San Carlos de Guatemala, adoptaron en la transición de clases a la virtualidad, situación que se presentó a raíz de la Pandemia del COVID-19. En cierto modo el papel y perfil del docente ha tenido cambios en las últimas décadas como consecuencia de las demandas de la sociedad del conocimiento aunadas a los avances de la tecnología que han aportado elementos para realizar una readecuación en la forma de desarrollar la docencia.

En ese sentido, la investigación permitió analizar los cambios que adoptaron los docentes para desarrollar la enseñanza a través de la virtualidad. Se desarrolló un estudio de enfoque cualitativo, con un 
diseño fenomenológico y alcance descriptivo. Las técnicas utilizadas para la recolección de información fueron entrevistas y la observación. Dentro de los resultados de la investigación se puede mencionar que, en la medida en que se llevó a cabo esta transición se realizaron cambios en la metodología para llevar a cabo el trabajo docente, se asumieron los desafíos en la organización y planificación de actividades utilizando herramientas virtuales que permitieran cubrir los contenidos de las clases. Finalmente, el papel del docente en la virtualidad requiere de capacitación para la planificación de actividades sincrónicas y asincrónicas.

\section{Abstract}

This article presents a description of the role and profile that the teachers of the Faculty of Economic Sciences of the University of San Carlos of Guatemala, had to adopt in the transition from classes to virtuality, a situation that was presented following the COVID-19 Pandemic. In a way, the role and profile of the teacher has changed in recent decades as a result of the demands of the knowledge society coupled with the advances in technology that have provided elements to carry out a readequation in the way teaching is developed.

In this sense, the research allowed to analyze the changes that teachers adopted to develop teaching through virtuality. A qualitative approach study was developed, with a phenomenological design and descriptive scope. The techniques used for information collection were interviews and observation. Among the results of the research, it can be mentioned that, to the extent that this transition was carried out, changes were made to the methodology to carry out the teaching work, the challenges in the organization and planning of activities were assumed using virtual tools that would allow to cover the contents of the classes. In conclusion, the role of the teacher in virtuality requires training for the planning of synchronous and asynchronous activities.

\section{Introducción}

El siglo XXI trajo consigo avances tecnológicos para las sociedades del mundo entero, cada vez se tiene más acceso a la información y a nuevos conocimientos, en lo que a educación se refiere, se han manifestado cambios en el proceso de enseñanza aprendizaje dejando
Palabras clave:

papel del docente, perfil del docente, metodología, planificación, organización.
Keywords: role of the teacher, teacher profile, methodology, planning, organization. 
por un lado el sistema tradicional de enseñanza. Las tecnologías de información y comunicación (TIC) son herramientas que permiten realizar una adecuación del contenido de los cursos, mediado a través de materiales pedagógicos que permitan alcanzar las competencias de los estudiantes (UNESCO, 1998).

A partir del desarrollo tecnológico, el docente en la educación superior ha implementado estrategias que permiten desarrollar sus clases de tal forma que, las herramientas digitales sirven como acompañamiento del proceso de enseñanza aprendizaje. Esto pone de manifiesto que el papel y perfil del docente demanda de una responsabilidad en la formación del aprendizaje autónomo, para la creación de nuevas competencias y habilidades para el desarrollo de la vida profesional de sus estudiantes (UNESCO, 1998).

Por otro lado, la situación de la educación superior en Guatemala según el censo del año 2018 refleja que únicamente el 4.56\% de la población cuenta con una formación profesional a nivel de licenciatura. Siendo la Universidad de San Carlos de Guatemala la única universidad estatal se ha visto en la necesidad de ampliar las carreras de acuerdo con las demandas del mercado laboral del país; en ese sentido estas acciones han requerido de una formación docente en la generación del conocimiento científico y tecnológico.

La educación como medida de prevención de la propagación de la pandemia del COVID-19 generó el cierre de los centros de educativos. En Guatemala se presentó el primer caso de COVID-19 el 13 de marzo de 2020, por lo que el Consejo Superior Universitario de la USAC acordó suspender las clases presenciales a partir del 16 de marzo de 2020, para resguardar la vida y la salud de los estudiantes y trabajadores, con ello la educación dio un giro de $180^{\circ}$, se presentaba una situación que no se tenía contemplada y se hizo necesario tomar medidas que permitieran continuar con la formación de los estudiantes.

En este contexto la Facultad de Ciencias Económicas de la Universidad de San Carlos de Guatemala asumió el reto de trasladar las clases presenciales a virtuales de tal forma que no se detuviera el proceso de enseñanza aprendizaje. Si bien es cierto, la modalidad presencial y virtual tienen diferencias en lo que se refiere a la forma de impartir y adquirir el conocimiento, a pesar de que la finalidad es lograr una calidad en la enseñanza, las metodologías empleadas son distintas. 
De tal forma que, la importancia del estudio se basó principalmente en describir cuál fue el papel y perfil que los docentes adoptaron en esa transición que demandó trasladarse de un salón de clase presencial a estar frente a una pantalla desarrollando una clase virtual, tomando en consideración que actualmente las tecnologías de información y comunicación juegan un papel crucial en el desarrollo de las actividades educativas, los docentes han tenido que valerse de estas como herramientas esenciales para el desarrollo de clases, asignación de tareas, evaluaciones entre otras.

Nadie estaba preparado para enfrentar la pandemia del COVID-19, por lo que es comprensible que se haya tenido que tomar medidas que se adapten a las necesidades de cada uno de los docentes, desde impartir una clase a través de una computadora de escritorio, laptop, tableta o teléfono celular. El objetivo del artículo es, describir el papel y perfil de los docentes del primer año de la jornada nocturna de la Facultad de Ciencias Económicas, Universidad de San Carlos de Guatemala en la transición de clases presenciales a virtuales en el año 2020.

\section{Materiales y métodos}

La metodología utilizada en el estudio es el enfoque cualitativo, con un diseño fenomenológico y alcance descriptivo, las técnicas utilizadas fueron la observación, la entrevista a profundidad y entrevistas estructuradas; es importante mencionar que los instrumentos utilizados previo al estudio, se aplicó el método de evaluación de coeficiente de validez de contenido de Hernández-Nieto, 2011 que valora el grado de acuerdo por juicio de expertos. En ese sentido, se trabajó con una muestra no probabilística de tipo homogénea con los docentes del primer año del Área Común de la Facultad de Ciencias Económicas, de la Universidad de San Carlos de Guatemala en el año 2020. 


\section{Resultados}

\section{Tabla 1}

Cambios que se llevaron a cabo en el papel y perfil del docente en los entornos virtuales

\begin{tabular}{|c|c|c|}
\hline Categorías & f & Evidencia \\
\hline Planificación & 13 & $\begin{array}{l}\text { "Una preparación más exhaustiva respecto del abordaje de los temas y la mejor forma } \\
\text { de presentarlos", "Preparar la clase de una forma más visual", "La forma de desarrollar } \\
\text { el contenido", "Adaptar el material”, "Cambios en la metodología de evaluación", } \\
\text { "Material didáctico", "Extensión de horarios de clase versus cantidad de contenidos", } \\
\text { "Reordenamiento de los temas para conformar un hilo conductor más adecuado", } \\
\text { "Cambios metodológicos", "El tiempo y la modalidad virtual”, "La preparación del curso } \\
\text { es diferente ya que hay que preparar el curso con ayuda de programas como Word, } \\
\text { Excel, PowerPoint, Powtoon", "La utilización de herramientas, entrega de tareas, formas } \\
\text { de integración de grupos", "Preparar más material didáctico en diferentes programas". }\end{array}$ \\
\hline Uso de tecnología & 7 & $\begin{array}{l}\text { "Uno de los principales cambios es equiparse tanto de tecnología cómo de internet", } \\
\text { "Tecnificarse más en el uso de las herramientas virtuales", "Tecnificación y uso de } \\
\text { tic's", "Clases con dispositivos", "Manejo de internet y plataformas que anteriormente } \\
\text { desconocía", "El uso de la tecnología virtual", "Grabar la totalidad de las sesiones", } \\
\text { "Crear formatos en Drive para hacer ejercicios en línea". }\end{array}$ \\
\hline Adaptación & 6 & $\begin{array}{l}\text { "Adquirir convicción y compromiso", "Adaptarme a la tecnología”, "Adaptación a la } \\
\text { tecnología y a la modalidad virtual", "Capacitación", "Aprender a utilizar herramientas } \\
\text { virtuales", "Cambios estratégicos en la planificación", "Método de enseñanza", } \\
\text { "Competencias digitales". }\end{array}$ \\
\hline Comunicación & 6 & $\begin{array}{l}\text { "Adecuada comunicación virtual”, "Los medios de comunicación con los estudiantes", } \\
\text { "Mayor comunicación extra aula con los estudiantes en asuntos del curso", "La normal, } \\
\text { a pesar de no estar unidos físicamente, "La comunicación ha sido aceptable", "Bastante } \\
\text { buena, Vía WhatsApp y video conferencias", "Muy buena. "Han sido más exigentes". }\end{array}$ \\
\hline
\end{tabular}

Fuente: elaboración propia con base a datos obtenidos en entrevistas realizadas.

Dentro de los principales cambios realizados en el papel del docente se resume que, la planificación de las clases virtuales demandó un ajuste de las actividades de tal forma que, se adecuó con el tiempo de clases sincrónicas y los contenidos a desarrollar, se utilizaron plataformas y herramientas virtuales que permitieron continuar con el proceso de enseñanza aprendizaje. Asimismo; los docentes manifestaron que se requirió de una preparación más exhaustiva para el desarrollo de la temática de los cursos, así como también la tecnificación en el uso de las Tics, lo cual requirió de capacitación para el manejo de las plataformas virtuales. 
Asimismo; se puede mencionar que, los cursos teóricos requirieron de una adecuación para atraer la atención de los estudiantes, por medio del uso de diversas plataformas de comunicación que permitieran la comprensión del contenido a desarrollar, se crearon formularios en Google Drive para hacer evaluaciones en línea y se implementó el uso de infografías para transmitir el mensaje de una manera más sencilla. Un aspecto a resaltar es la extensión de horarios en relación con los contenidos, lo cual se relaciona con el tiempo del período de clase, en el cual los docentes manifestaron que los estudiantes demandan de más tiempo para resolver dudas de los temas desarrollados en la clase.

En lo que se refiere al perfil, del docente se evidenció que es indispensable contar con las competencias digitales que permitan gestionar una metodología apoyada con el uso de la tecnología. En lo que referente a la comunicación con los estudiantes, se utilizaron los medios tecnológicos como redes sociales, correo electrónico y WhatsApp, a pesar de no estar físicamente se logró una respuesta favorable, fue más fluida y en algunos casos los estudiantes fueron más exigentes, eso debido a que, tenían la posibilidad de realizar consultas en cualquier momento, lo cual requería de más tiempo por parte del docente para poderles atender.

\section{Discusión}

El estudio muestra que los docentes asumieron el reto en este cambio tan repentino hacia la virtualidad, sin embargo, reconocen la debilidad que persiste en las competencias digitales virtuales para el desarrollo del proceso de enseñanza aprendizaje. Como lo manifiesta Rederecker (2020) las competencias digitales se pueden manifestar de acuerdo con los cambios que demanda la sociedad del conocimiento, en el ejercicio de competencias cada vez más exigentes, en la medida en la que los estudiantes se deselvuelven a través de la virtualidad así también es necesario que los docentes desarrollen su propia competencia digital.

En ese sentido, potencializar las competencias digitales depende de la capacidad de los docentes para estructurar los contenidos con la integración de las tecnologías de información y comunicación. La investigación reflejó que se han presentado cambios en el papel del docente, dentro de los cuales se puede mencionar la planificación más exhaustiva para desarrollar los contenidos desde la virtualidad, con el 
apoyo de herramientas tecnológicas como Word, Excel, PowerPoint, Powtoon, Google drive, Classroom, Google Meet y Zoom.

Por otro lado, los estudiantes cada vez son más exigentes, requieren de la dirección y acompañamiento del docente para lograr un conocimiento más reflexivo, lo cual amerita que el docente realice la función de facilitador y organice las actividades de tal forma que, se despierte el interés y se mantenga la atención de los estudiantes. Los estudiantes se han adaptado de forma positiva, según se logró determinar en las observaciones realizadas a las clases virtuales, el docente promueve la participación de los estudiantes con actividades didácticas; sin embargo, no todos habilitan su cámara, lo cual genera cierta incertidumbre en el docente puesto que no se tiene la certeza de que el estudiante esté atento al desarrollo de la temática.

Las sesiones sincrónicas se desarrollan por medio de Google Meet en el horario habitual de la clase y se cumple con los contenidos programáticos al igual que las clases presenciales. De igual manera como lo manifiesta en su estudio Miguel (2020), que la transición a la virtualidad fue un ajuste de emergencia en donde se trasladó lo presencial a lo virtual; mismos horarios, mismos contenidos y retroalimentación. Asimismo UNESCO dice que "el profesorado se ha visto desafiado a ubicar resoluciones creativas e innovadoras, actuando y aprendiendo sobre la marcha, demostrando capacidad de adaptabilidad y flexibilización de los contenidos y diseños de los cursos para el aprendizaje en las distintas áreas" (2020.p. 21).

El cambio tan repentino a la virtualidad como consecuencia de la pandemia del COVID-19, tomó por sorpresa a todos, no había una preparación previa para atender este tipo de eventualidades; por lo que es comprensible que los docentes tomaran medidas pedagógicas que se adaptaran a sus necesidades, con el objetivo de cumplir con el papel de mediador del aprendizaje desde entornos virtuales, las cuales fueron perfeccionando a través de la práctica.

Para finalizar, se puede concluir que, ante la transición de clases presenciales a virtuales como consecuencia de la pandemia del COVID-19, se manifestaron cambios en el papel del docente, los cuales van enfocados en la planificación y metodología para el desarrollo de las clases sincrónicas, preparación de actividades con el apoyo 
de herramientas tecnológicas, convertirse en un guía-orientador y la forma de evaluar los conocimientos adquiridos por los estudiantes.

En lo que se refiere al perfil del docente sobresale el desarrollo de competencias para el manejo de las herramientas virtuales de aprendizaje y la capacidad de mantener la comunicación con los estudiantes a través de los medios tecnológicos como redes sociales, correo electrónico y WhatsApp.

Por lo antes mencionado, se recomienda diseñar la planificación y programas de contenidos académicos para el desarrollo del proceso de enseñanza aprendizaje desde la virtualidad, asimismo; se recomienda la capacitación constante para los docentes en las tecnologías de información y comunicación para generar nuevas formas de enseñanza y aprendizaje.

\section{Agradecimientos}

El estudio fue realizado gracias al apoyo brindado por la Facultad de Ciencias Económicas de la Universidad de San Carlos de Guatemala, el asesor de tesis Maestro José Bidel Méndez y la Doctora Walda Flores Luin docente del curso de tesis.

\section{Referencias}

Miguel, J. (24 de 7 de 2020). La educación superior en tiempos de pandemia: una visión desde dentro del proceso formativo. Revista Latinoamericana de Estudios Educativos, L. doi:https://doi.org/10.48102/rlee.2020.50.ESPECIAL.95

Rederecker, C. (2020). Marco europeo para la competencia digital de los educadores. DigCompEdu. (O. d. Europea, Ed.) 95 . doi:10.2760/15977

UNESCO. (1998). Conferencia Mundial sobre la Educación Superior en el Siglo XXI: Visión y Acción. Recuperado el 6 de agosto de 2020, de http://www.iesalc.unesco. org/ess/index.php/ess $3 /$ article/view/171/162 
Villafuerte, J., Bello, J., Pantalón, Y., \& Bermello, J. (20 de 4 de 2020). Rol de los docentes ante la crisis del COVID-19, una mirada desde el enfoque humano. Revista Electrónica Formación y Calidad Educativa, 17. Recuperado el 2 de abril de 2021, de http://www. refcale.uleam.edu.ec/index.php/refcale/article/view/3214

\section{Sobre el autor}

Licenciada en Administración de Empresas, egresada de la Facultad de Ciencias Económicas de la Universidad de San Carlos de Guatemala, docente de Técnicas de Investigación; Documental y Recursos Económicos de Centro América, en el Área Común de la Facultad de Ciencias Económicas de la Universidad de San Carlos de Guatemala, estudiante de la Maestría en Docencia Universitaria de la Escuela de Estudios de Posgrado, Facultad de Humanidades de la Universidad de San Carlos de Guatemala.

Copyright (c) Yeny Audelí Orellana Linares

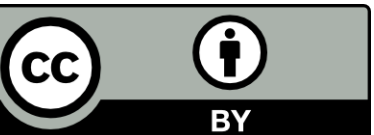

Este texto está protegido por una licencia Creative Commons 4.0.

Usted es libre para compartir, copiar y redistribuir el material en cualquier medio o formato y adaptar el documento, remezclar, transformar y crear a partir del material para cualquier propósito, incluso comercialmente, siempre que cumpla la condición de atribución: usted debe reconocer el crédito de una obra de manera adecuada, proporcionar un enlace a la licencia, e indicar si se han realizado cambios. Puede hacerlo en cualquier forma razonable, pero no de forma tal que sugiera que tiene el apoyo del licenciante o lo recibe por el uso que hace. 